\title{
Measurements of Particle Production, Underlying Event and Double Parton Interactions at the LHC
}

Jan Kuechler*†

Bergische Universität Wuppertal

E-mail: jan.kuechler@cern.ch

Various features of quantum chromodynamics (QCD) are an important part of the phenomenology of the events at hadron colliders, such as the LHC, including soft and non-perturabative features. In order to constrain the parameters of models of soft QCD, diverse measurements are provided by the ALICE, ATLAS and CMS collaborations. Measurements of particle production, underlying event and double parton interactions are presented. In general, reasonable agreement between the measured data and the models is found, but discrepancies hint at the need for a better description.

Fourth Annual Large Hadron Collider Physics

13-18 June 2016

Lund, Sweden

* Speaker.

${ }^{\dagger}$ On behalf of the ALICE, ATLAS and CMS collaborations. 


\section{Introduction}

Various features of quantum chromodynamics (QCD) are an important part of the phenomenology of the events at hadron colliders, such as the LHC. Such effects also include soft and nonperturabative features. They contribute to the modelling of additional proton-proton interactions in one bunch crossing (pile-up) and multiple parton-parton interactions in one proton-proton collision, as well as the underlying event. Due to the non-pertubative nature of low energy QCD only phenomenological models exist to describe the behaviour of these effects. In order to constrain the input parameters of these models, diverse measurements of these soft-QCD effects are needed.

The ALICE [1], ATLAS [2] and CMS [3] collaborations at the LHC have published a large set of measurements at various centre-of-mass energies.

\section{Particle Production in Minimum Bias Measurements}

Inclusive charged particle measurements, so-called minimum bias measurements, probe softQCD interactions with a low momentum transfer. The understanding of these interactions plays a crucial role in the modelling of pile-up interactions. The measurements at $\sqrt{s}=13 \mathrm{TeV}$ are based on tracks, which are corrected for detector effects and unfolded to particle level $[4,5,6]$.

Figure 1 shows the measured distributions of the transverse momentum $p_{\mathrm{T}}$, the pseudo-rapidity $\eta$ and the charged-particle multiplicity. The EPOS generator [7] with the LHC tune, which is based on a parton-level Gribov-Regge theory as well as hydrodynamic effects and is typically used in astroparticle physics, shows a very good description of the data. The same can be seen for the Monash tune of the Pythia8 generator [8]. The HeRwig++ generator [9] is tuned to describe underlying event behaviour and shows strong discrepancies compared to the minimum bias data.
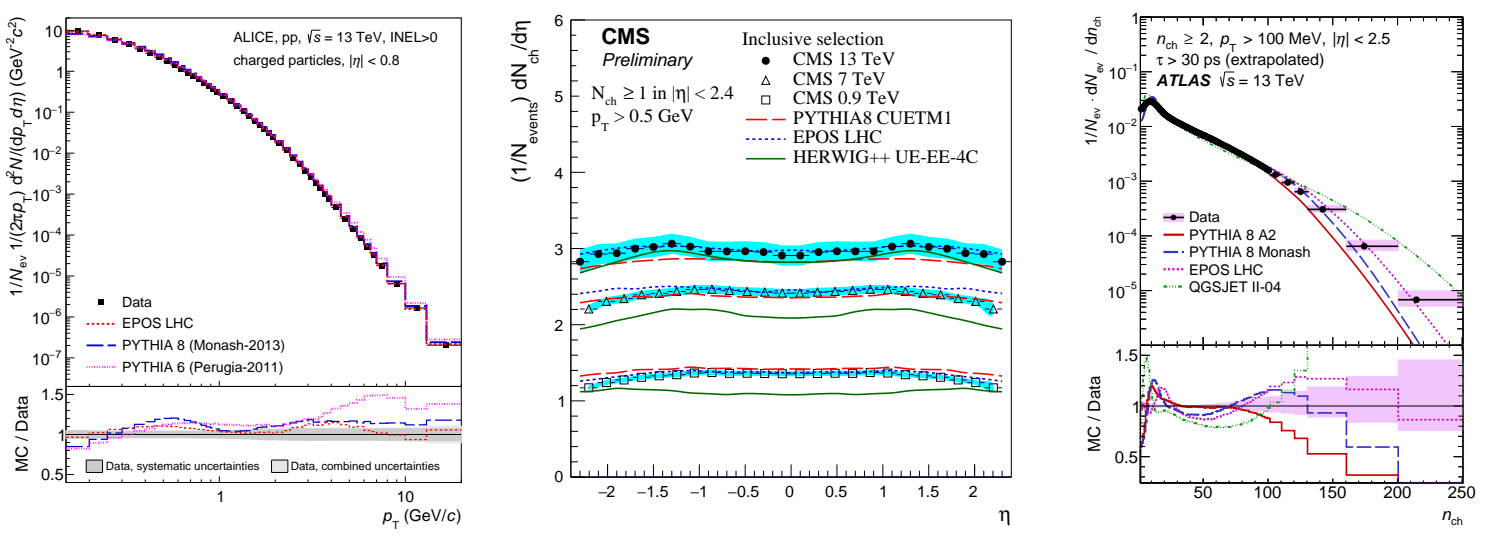

Figure 1: Distribution of the $p_{\mathrm{T}}$ (left, ALICE [4]), pseudo-rapidity $\eta$ (center, CMS [6]) and charged-particle multiplicity (right, ATLAS [5]) of inclusive charged particles compared to various model predictions of different generators.

The rise of the mean charged-particle multiplicity, measured in the central $\eta$ region, as a function of the centre-of-mass energy is shown in Figure 2. The general behaviour is well described by all models considered. Again, the EPOS generator provides the best description. 


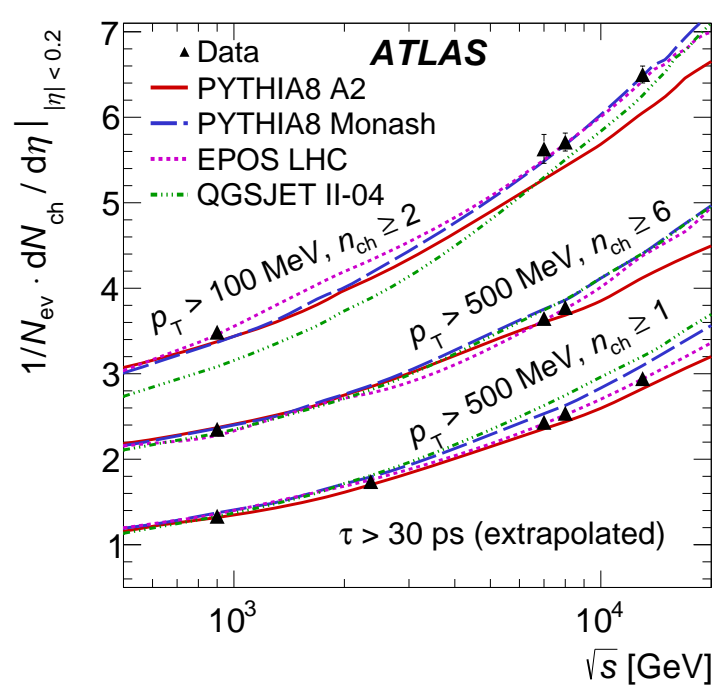

Figure 2: Charged-particle multiplicity in the central rapidity region, $|\eta|<0.2$, as a function of the centreof-mass energy in various regions of phase-space, compared to different model predictions as measured by ATLAS [5].

In addition to the inclusive measurements, the ALICE collaboration measured the rate of strange particle production, i.e. the strange particle yield relative to the charged pion yield, as a function of the charged-particle multiplicity [10]. The strange hadron rate is shown in Figure 3, for proton-proton, proton-lead and lead-lead collisions. While generally the rates at low multiplicity proton-proton collisions are reasonably well predicted by theory calculations, the intermediate rise in the strangeness production is not modelled by any of the simulations, especially for the $\Omega$ rate. This measurement shows a clear deficit in the currently used tunes for both the PYTHIA and the Epos model.

\section{The Underlying Event}

The underlying event (UE) encompasses the activity in a proton-proton scattering which occurs in addition to the parton-parton hard scattering. These are initial and final state radiation, multiple, soft parton interactions (MPI) and the proton remnants. Just as for inclusive particle production, only phenomenological models exists to describe the UE, which have to be tuned based on measurements. Due to its contribution to a proton-proton interaction, the UE can be crucial for precision measurements at the LHC.

Typical measurements of the UE require the hard process to be separated from the remnants. In hard jet production, this can be done by splitting the particles in the event into three regions in $|\Delta \phi|$ to the leading object. The toward region, defined as $|\Delta \phi|<60^{\circ}$, contains the particles close to the leading object, which can be strongly influenced by the hard scattering. The away region, $|\Delta \phi|>120^{\circ}$, is dominated by the recoil of the leading object. The transverse regions, with $60^{\circ}<|\Delta \phi|<120^{\circ}$, are assumed to be unaffected by the hard scattering and are therefore sensitive to the UE activity. 


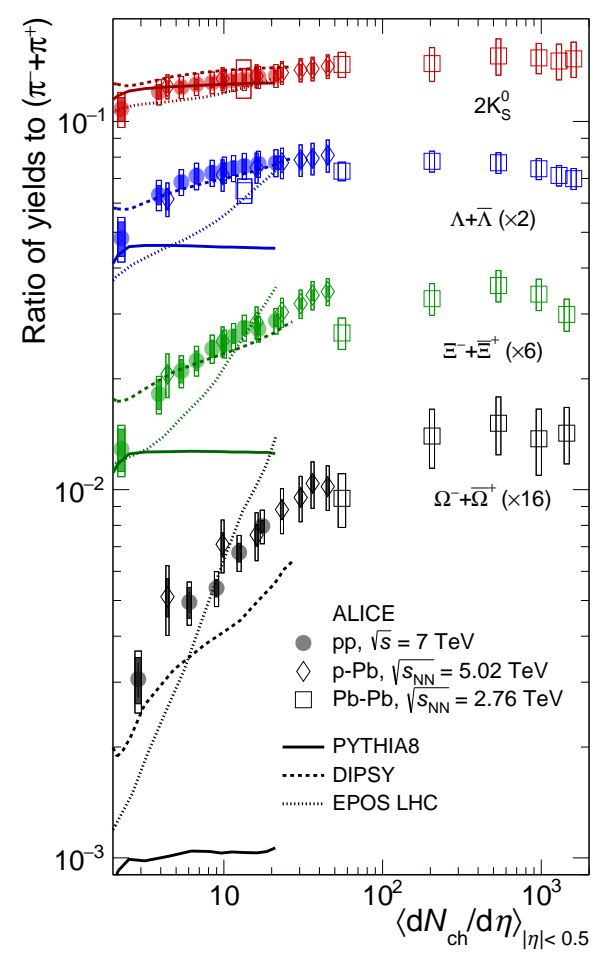

Figure 3: Measurement of the strange hadron production rate as a function of the charged-particle multiplicity by ALICE [10].

Alternative methods require a colour-neutral object, like a leptonically decaying $Z$ boson, in order to provide a trigger signature. As the $Z$ boson and its decay products in this case do not interact via QCD, this allows for a clean measurement of the UE.

The ATLAS collaboration has published a detector-level measurement [11] of the UE at $\sqrt{s}=13 \mathrm{TeV}$. The analysis closely follows the minimum bias measurement, but the results are not unfolded to particle level. Figure 4 shows the mean energy density and the mean particle density in events with a leading track of $p_{\mathrm{T}}>1 \mathrm{GeV}$ and $p_{\mathrm{T}}>5 \mathrm{GeV}$, respectively. In the transverse region, most of the simulations agree within $10 \%$ with the measured data. The level of agreement is similar for the energy and the particle density. At low leading track $p_{\mathrm{T}}$ the HERWIG++ EE5 tune [12] shows larger discrepancies, as this region is more similar to inclusive charged particle production as measured in the minimum bias analyses. At higher leading track $p_{\mathrm{T}}$, the minimum bias tune A2 [13] for PYTHIA8 does not agree well with the measurement.

The CMS collaboration published a measurement of the $\mathrm{UE}$ at $\sqrt{s}=13 \mathrm{TeV}$ that is fully corrected and unfolded to particle level [14]. In this measurement, both single tracks as well as jets are considered as the leading object, with separate distributions for both cases. The observables are the mean particle density and the mean energy density of charged particles with $p_{\mathrm{T}}>0.5 \mathrm{GeV}$ and $|\eta|<2.0$ in the event. In this measurement, several effective configurations of the transverse region are considered. transMAX and transMIN label the regions of phase space with $60^{\circ}<|\Delta \phi|<120^{\circ}$ to the leading object with the higher, or lower, density, respectively. The former region is more sensitive to the additional QCD radiation in the event, while the latter one contains the effects of 

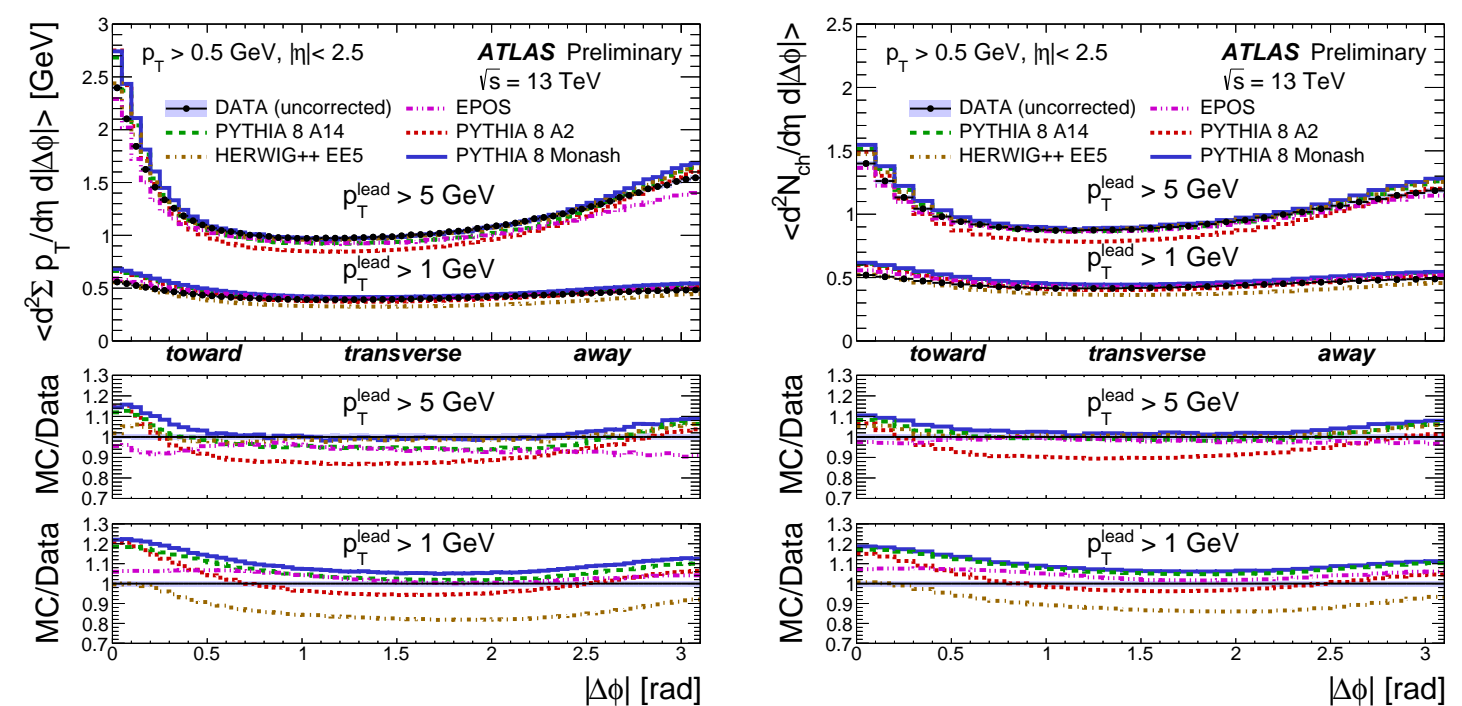

Figure 4: Detector-level measurements by ATLAS of the mean energy density (left) and the mean particle density (right) as a function of the $|\Delta \phi|$ to the leading charged-particle in the event, in comparison to different model predictions [11].

the generally softer multiple parton scattering. These effects also enter the MAX region, but are small compared to the radiation.
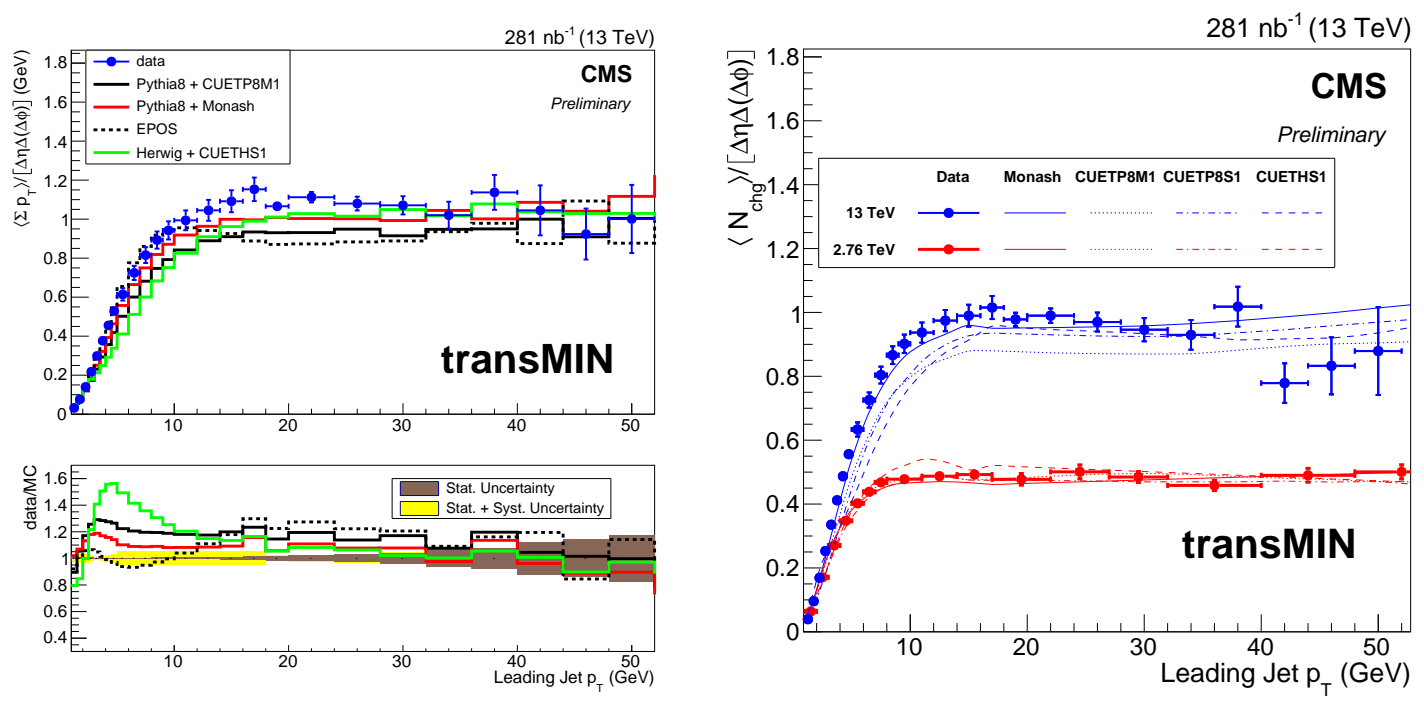

Figure 5: Particle level measurements by CMS of the mean energy density (left) and the mean particle density (right) as a function of the leading jet $p_{\mathrm{T}}[14]$.

Figure 5 shows the measurements in the transMIN region as a function of the leading jet $p_{\mathrm{T}}$. Overall the data is reasonably described by the models, with the best agreement found in the comparison to the Pythia8 Monash tune. While Epos describes the low $p_{\mathrm{T}}$ region well, the model shows larger deviations at higher leading jet $p_{\mathrm{T}}$. In comparison to the measurement at 
$\sqrt{s}=2.76 \mathrm{TeV}$, the particle density increases strongly with the centre-of-mass energy. The overall level of agreement of the simulation is worse at $\sqrt{s}=13 \mathrm{TeV}$.

A measurement of the event shapes in leptonic $Z$ boson events at $\sqrt{s}=7 \mathrm{TeV}$ is published by ATLAS [15]. As the $Z$ boson and its decay products do not interact via the strong interaction, all charged particles excluding the two leptons from the $Z$ decay are considered in the observables. The measurements are corrected for detector effects and pile-up, and unfolded to particle level for charged particles with $p_{\mathrm{T}}>0.5 \mathrm{GeV}$ and $|\eta|<2.5$.
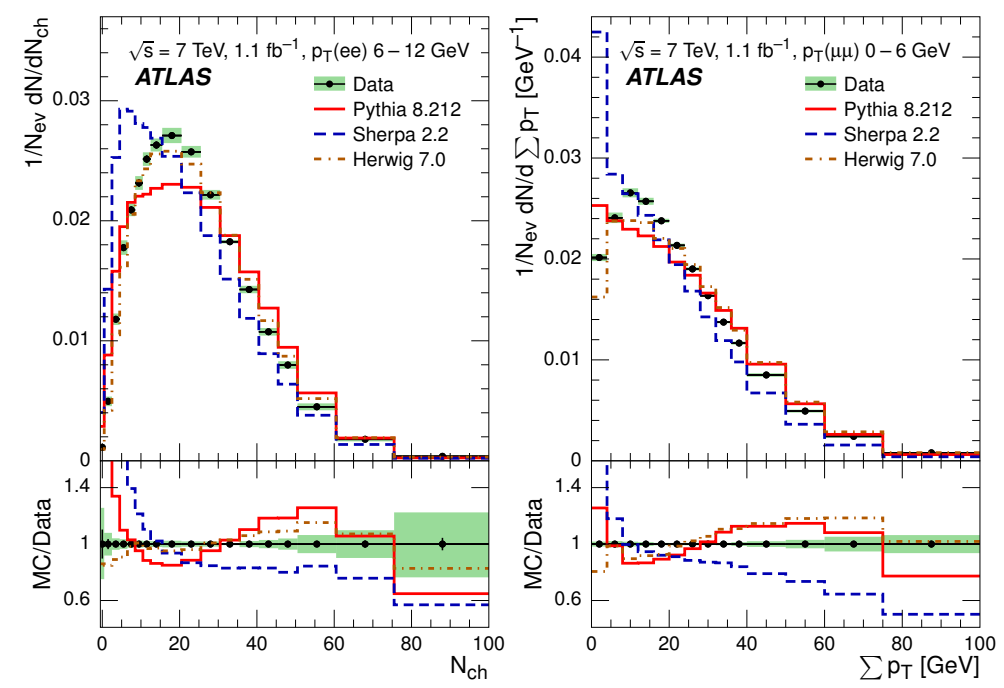

Figure 6: Charged particle multiplicity (left) and the scalar sum of the charged particle transverse momenta (right) for low $p_{\mathrm{T}}$ leptonic $Z$ boson events, measured by ATLAS [15].

In the measurement, inclusive event shape variables like the transverse thrust and the spherocity were found to show a good agreement between data and simulations. For lower $Z$ boson $p_{\mathrm{T}}$, the charged particle multiplicity, $\mathrm{N}_{\mathrm{ch}}$, and strongly correlated observables like the scalar sum of the charged particle transverse momenta, $\sum \mathrm{p}_{\mathrm{T}}$, show sizeable discrepancies between the considered models and the data. Figure 6 shows two exemplary distributions. The best description is given by the HERWIG 7.0 generator, which shows reasonable agreement in the charged particle multiplicity. Nonetheless, for the $\sum \mathrm{p}_{\mathrm{T}}$ distribution significant deviations are observed.

\section{Double Parton Interactions}

Double parton interactions (DPI, also DPS for double parton scattering) are the occurrence of two hard parton-parton interactions in one proton-proton interaction. The cross section for the DPI process $p p \rightarrow A+B$, where both $A$ and $B$ are independent parton-parton interactions, is given phenomenologically by

$$
\sigma_{A+B}^{\mathrm{DPI}}=\frac{1}{1+\delta_{A B}} \frac{\sigma_{A} \sigma_{B}}{\sigma_{\mathrm{eff}}}
$$

where $\sigma_{A}$ and $\sigma_{B}$ are the single parton interaction (SPI, SPS) cross sections for the processes $A$ and $B$, respectively. The term $\frac{1}{1+\delta_{A B}}$ is a symmetry factor, which is $\frac{1}{2}$ for identical processes $A=B$, or 1 , 
otherwise. The "effective cross-section" $\sigma_{\text {eff }}$ characterises the transverse area of the proton-proton interaction. It is assumed to be independent of the processes $A$ and $B$, and measurements so far show no indication of a centre-of-mass dependence, being consistent with a value of $\sigma_{\text {eff }} \simeq 15 \mathrm{mb}$. This description is based on the assumption that the processes $A$ and $B$ are uncorrelated.

The CMS collaboration published a search for DPI in same-sign $W W$ events, in the $W^{ \pm} W^{ \pm} \rightarrow$ $\mu^{ \pm} \mu^{ \pm}+2 v$ channel. Due to the low cross section of the SPI same-sign contribution, this analysis profits from a high signal to background ratio. The separation of the double- and single-parton interactions faces several challenges. In order to exploit the differences in the angular correlations from the leptons, which differ because the DPI processes are uncorrelated, a boosted decision tree (BDT) is trained. Additional information is provided by the lower boost of the $W$ bosons in DPI events, in which the bosons do not recoil against each other.
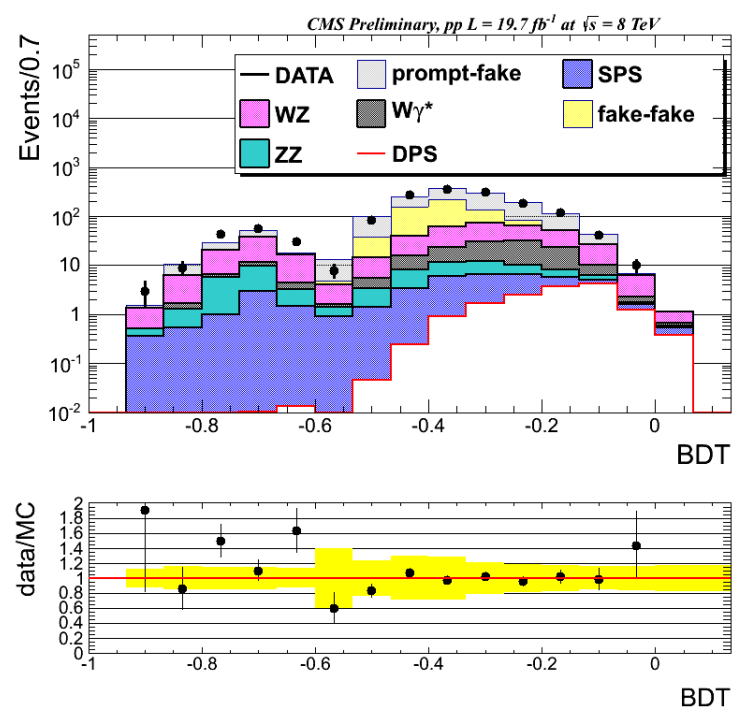

Figure 7: Distribution of the BDT output, used to separate the DPI signal and the various backgrounds in the CMS analysis [16].

Figure 7 shows the distribution of the BDT output used to separate the DPI signal from the various backgrounds. Based on this, the 95\% CL limits on the cross section for DPI $W W$ production is $\sigma_{W W}^{\mathrm{DPI}}<1.12 \mathrm{pb}$, with an expected limit of $1.18 \mathrm{pb}$. This translates into a lower limit on the effective cross section of $\sigma_{\text {eff }}>5.91 \mathrm{mb}$, consistent with previous measurements.

In an alternative analysis by CMS [17], a measurement of events with two $b$-tagged and two light jets is performed. Parts of the phase space, in which the two light jets and the two $b$-jets are back to back, are sensitive to DPI. The measurement is based on low pile-up data at $\sqrt{s}=7 \mathrm{TeV}$ from 2010 .

In general, good agreement was found between the measurement and the simulation, in the differential distributions of the jet $p_{\mathrm{T}}$ and $\eta$, as can be seen in Figure 8 (left). The mean jet $p_{\mathrm{T}}$ balance, defined as

$$
\Delta^{\mathrm{rel}} p_{\mathrm{T}}=\frac{\left|\vec{p}_{\mathrm{T}}^{\mathrm{jet} 1}+\vec{p}_{\mathrm{T}}^{\mathrm{jet}}\right|}{\left|\vec{p}_{\mathrm{T}}^{\mathrm{jet} 1}\right|+\left|\vec{p}_{\mathrm{T}}^{\mathrm{jet} 2}\right|},
$$


for the $b$-tagged jets, is not well reproduced by any model, as shown in Figure 8 (right). At small $\Delta^{\text {rel }} p_{\mathrm{T}}$, where the two jets are in balance, the measurement is sensitive to hard DPI, while the models are tuned to soft MPI as found in UE measurements. The analysis concludes, that the description of both soft MPI and hard DPI in the same model is difficult and not achieved.
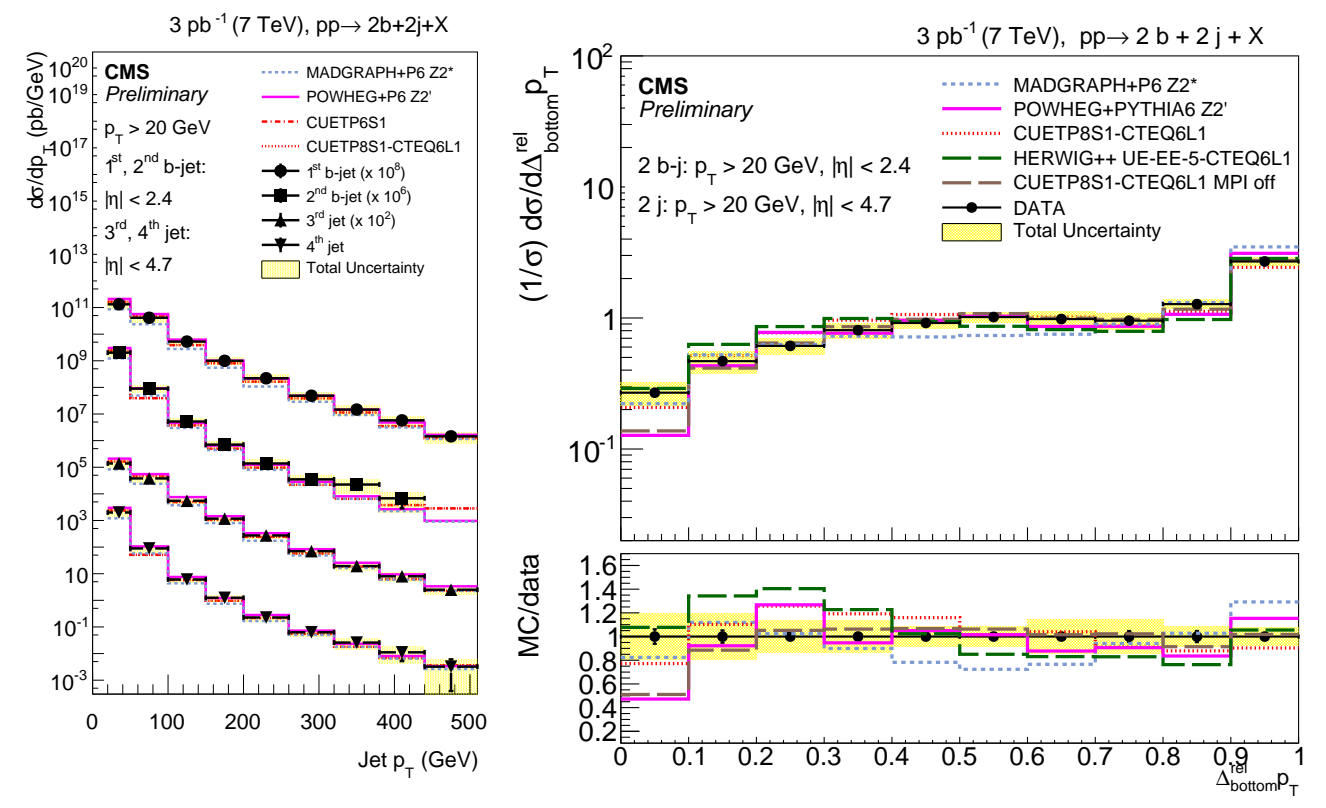

Figure 8: Measurement by CMS of the differential cross section of two $b$-jet plus two light jet production as a function of the jet $p_{\mathrm{T}}$ (left) and as a function of the relative $p_{\mathrm{T}}$ difference of the bottom jets (right) [17].

The ATLAS collaboration has measured the DPI cross section of four jet events in the low pileup data of 2010 at $\sqrt{s}=7 \mathrm{TeV}$ [18]. In this measurement, two classes of signal event topologies are considered. In the complete DPS case (cDPS) two of the four leading jets stem from one partonparton interaction and the other two jets from the other interaction. In the case of semi-complete DPS (sDPS), only one of the four leading jets is from one interaction, while the other three jets stem from the other parton-parton interaction, including QCD radiation.

In order to separate the two signal classes from SPS four jet events, a neural network is used. Sensitive inputs are mainly the $p_{\mathrm{T}}$ balance of the leading jets and the angular separations. While the SPS and sDPS models are taken from simulation, the cDPS signal is modelled by overlaying di-jet events from data. This removes any dependence on the modelling of these events in the simulation. Figure 9 shows the jet $p_{\mathrm{T}}$ balance of the third and fourth leading jet. The neural network is trained to classify the events into three categories: SPS, sDPS and cDPS. The sum of the outputs is normalised to one, $\xi_{\mathrm{SPS}}+\xi_{\mathrm{sDPS}}+\xi_{\mathrm{cDPS}}=1$, in order to allow each value to be interpreted as a probability.

Figure 10 shows the resulting measurement compared to the models. Although the jet $p_{\mathrm{T}}$ balance is a powerful variable to discriminate between DPS and SPS, the cDPS model is orders of magnitudes below the SPS contribution in any bin. In the neural network probabilities however, the bins with the highest cDPS probability are dominated by the cDPS model. This allows the cross section for the DPI production of four jet events to be extracted using a two-dimensional template 


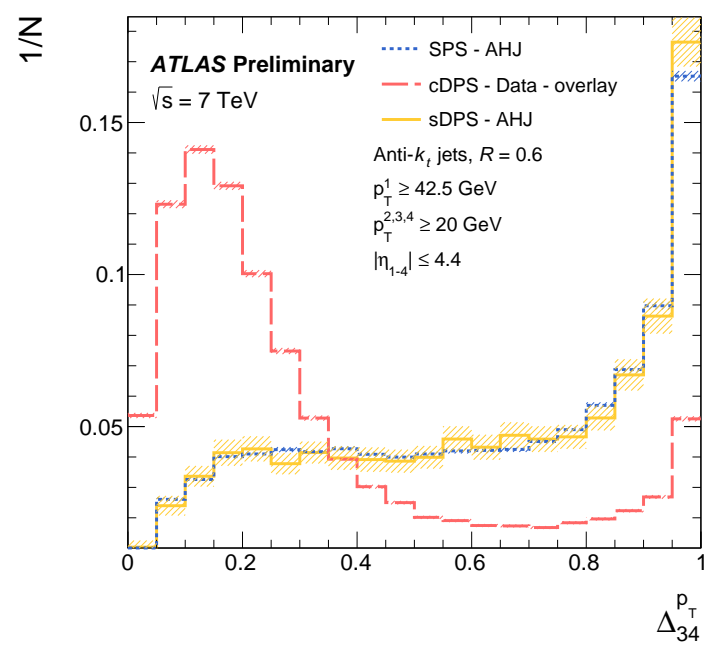

Figure 9: Distribution of the relative $p_{\mathrm{T}}$ difference of the third and fourth leading jet for DPS and SPS events in the ATLAS analysis [18].
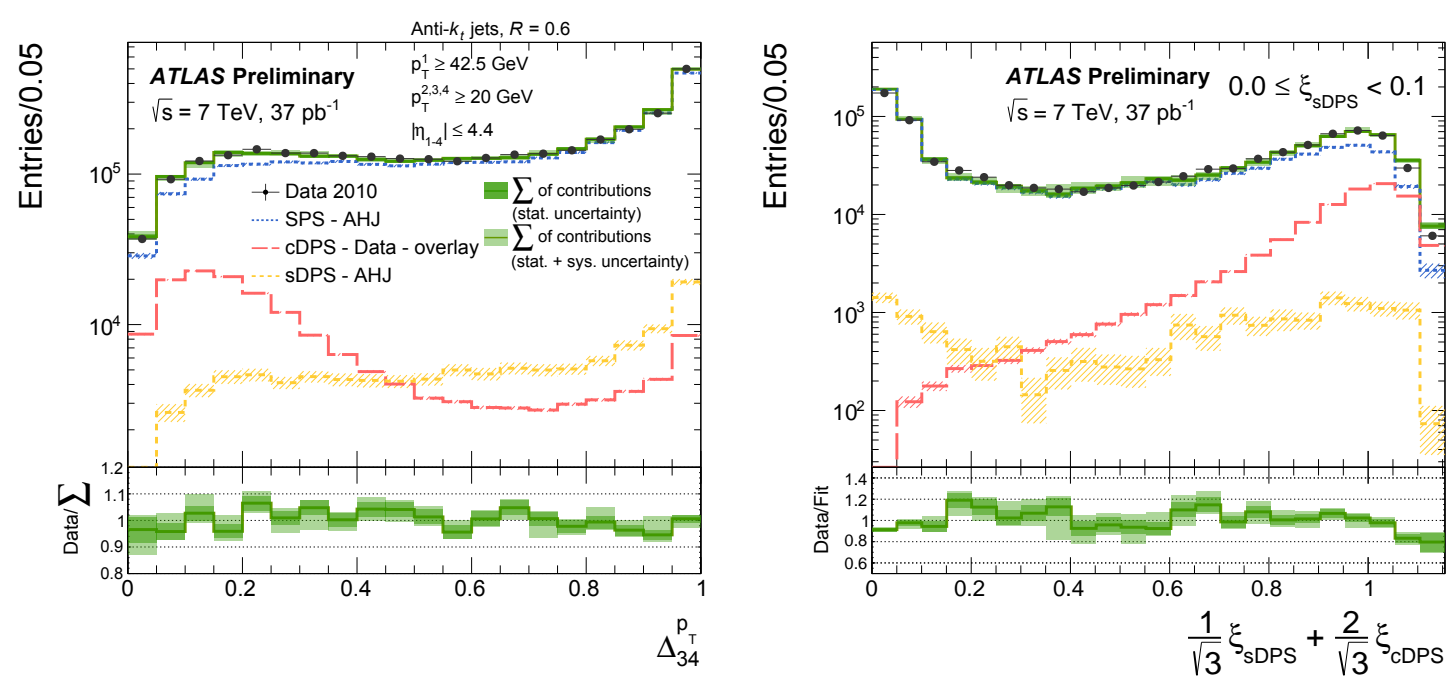

Figure 10: Measurement by ATLAS of the relative $p_{\mathrm{T}}$ difference of the third and fourth leading jet (left) and the normalised sum of the sDPS and cDPS probabilities for $0.0<\xi_{\text {SPS }}<0.1$ (right), as obtained by the neural network [18].

fit to the neural network output. From this, the parameter $\sigma_{\text {eff }}$ can be derived,

$$
\sigma_{\text {eff }}=16.1_{-1.5}^{+2.0}(\text { stat. })_{-6.8}^{+6.1} \text { (syst.) } \mathrm{mb} \text {. }
$$

This result is compared to previous measurements in Figure 11. Due to the complicated nature of these measurements the uncertainties are large, and as such, no dependence on the centre-of-mass energy can be observed. 


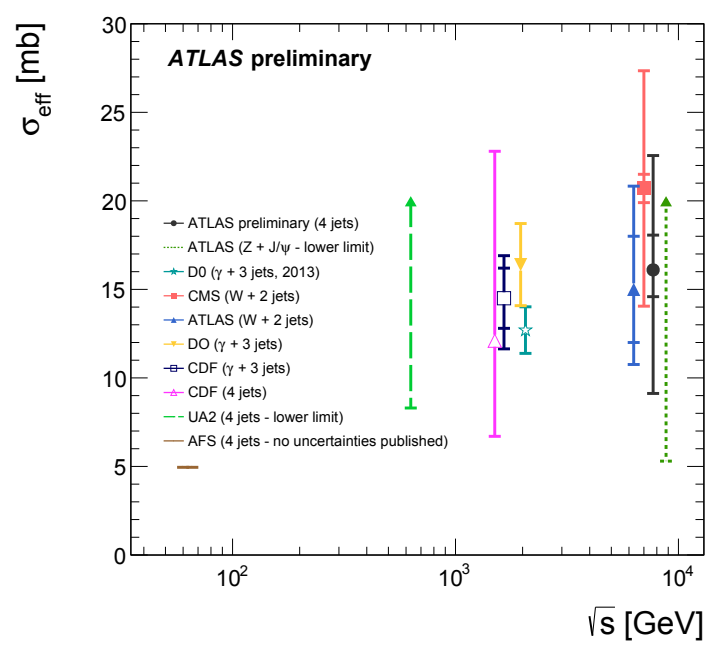

Figure 11: Measurements of the effective proton cross section, $\sigma_{\text {eff }}$ as a function of the centre-of-mass energy (ATLAS, [18]).

\section{Conclusion}

Measurements of particle production, underlying event and double parton interactions from the ALICE, ATLAS and CMS collaborations are presented. As the soft, non-pertubative region of QCD is only described by phenomenological models, these measurements provide important input for the validation and improvement of the modelling. This is important, as these types of interactions, together with the various other features of QCD, can play a crucial role in high precision measurements and as the background to searches for new physics.

In general, reasonable agreement between the measured data and the simulation is found. Discrepancies in measurements highlight the need for better description. For this, further tuning, but also refinement of the phenomenological models is needed.

\section{References}

[1] ALICE Collaboration, K. Aamodt et al, The ALICE experiment at the CERN LHC, JINST 3 (2008) S08002.

[2] ATLAS Collaboration, The ATLAS Experiment at the CERN LHC, JINST 3 (2008) S08003.

[3] CMS Collaboration, The CMS experiment at the CERN LHC, JINST 3 (2008) S08004.

[4] ALICE Collaboration, J. Adam et al, Pseudorapidity and transverse-momentum distributions of charged particles in proton-proton collisions at $\sqrt{s}=13 \mathrm{TeV}$, Physics Letters B 753 (2016): 319-329.

[5] ATLAS Collaboration, Charged-particle distributions at low transverse momentum in $\sqrt{s}=13 \mathrm{TeV}$ pp interactions measured with the ATLAS detector at the LHC, hep-ex/1606.01133.

[6] CMS Collaboration, Measurement of pseudorapidity distributions of charged particles in proton-proton collisions at $\sqrt{s}=13 \mathrm{TeV}$, CMS-PAS-FSQ-15-008, cds.cern.ch/record/2145373. 
[7] S. Porteboeuf, T. Pierog and K. Werner, Producing Hard Processes Regarding the Complete Event: The EPOS Event Generator, hep-ph/1006.2967.

[8] , T. Sjöstrand, S. Mrenna and P. Z. Skands, A Brief Introduction to PYTHIA 8.1, Comput. Phys. Commun. 178 (2008) 852-867.

[9] M. Bähr et al., Herwig++ physics and manual, Eur. Phys. J. C 58 (2008) 639.

[10] ALICE Collaboration, J. Adam et al, Multiplicity-dependent enhancement of strange and multi-strange hadron production in proton-proton collisions at $\sqrt{s}=7 \mathrm{TeV}$, hep-ex/1606.07424.

[11] ATLAS Collaboration, Detector level leading track underlying event distributions at 13 TeV measured in ATLAS, ATL-PHYS-PUB-2015-019, cds.cern.ch/record/2037684.

[12] S. Gieseke, C. Rohr, and A. Siodmok, Colour reconnections in Herwig++, Eur. Phys. J. C 72 (2012) 2225.

[13] ATLAS Collaboration, Further ATLAS tunes of Pythia6 and Pythia 8, ATL-PHYS-PUB-2011-014, cds.cern.ch/record/1400677.

[14] CMS Collaboration, Underlying event measurements with leading particles and jets in pp collisions at $\sqrt{s}=13 \mathrm{TeV}$, CMS-PAS-FSQ-15-007, cds.cern.ch/record/2104473.

[15] ATLAS Collaboration, Measurement of event-shape observables in $Z \rightarrow \ell^{+} \ell^{-}$events in pp collisions at $\sqrt{s}=7 \mathrm{TeV}$ with the ATLAS detector at the LHC, Eur. Phys. J. C (2016) 76: 375.

[16] CMS Collaboration, Double Parton Scattering cross section limit from same-sign W bosons pair production in di-muon final state at LHC, CMS-PAS-FSQ-13-001, cds.cern.ch/record/2103756.

[17] CMS Collaboration, Studies of 2 b-jet +2 jet production in proton-proton collisions at $7 \mathrm{TeV}$, CMS-PAS-FSQ-13-010, cds.cern.ch/record/2012675.

[18] ATLAS Collaboration, Study of hard double parton scattering in four-jet events in pp collisions at $\sqrt{s}=7 \mathrm{TeV}$ with the ATLAS experiment at the LHC, ATLAS-CONF-2015-058, cds.cern.ch/record/2108894. 